\title{
On the Marzke-Wheeler and Desloge Constructions
}

\author{
Alan Macdonald \\ Department of Mathematics \\ Luther College, Decorah, IA 52101, U.S.A. \\ macdonal@luther.edu
}

January 5, 2008

\begin{abstract}
There is no indication of time dilation of clocks or of length contraction of rods in Marzke and Wheeler's clock or in Desloge's metrosphere.
\end{abstract}

The nonmetric structure of a spacetime describes events, light rays, and free particles insofar as possible without reference to measurements by clocks or rods, i.e., without reference to the metric structure of the spacetime. Desloge, ${ }^{1}$ improving the work of Marzke and Wheeler, ${ }^{2}$ defines, using only the nonmetric structure of Minkowski spacetime $\mathcal{M}$, his "metrosphere". The metrosphere contains entities which behave just as "actual" clocks and rods behave in $\mathcal{M}$.

It is interesting that this can be done. But one must be cautious when interpreting Desloge's statement "the definition of the metrosphere leads to the usual kinematic results of special relativity". As examples of such kinematic results, he cites time dilation and length contraction. The purpose of this note is to point out that, despite the metrosphere, the nonmetric structure of Minkowski spacetime contains no hint of time dilation of "actual" clocks or length contraction of "actual" rods. It is essential to understand this when assessing the implications of the work of Marzke and Wheeler and of Desloge. It was understood by Reichenbach long ago. ${ }^{3}$ Indeed, this is a central point of his work. But perhaps a simple direct demonstration of this without all of Reichenbach's considerable machinery would be useful.

Call the clocks and rods of the metrosphere metroclocks and metrorods, and "actual" clocks and rods $\mathcal{M}$-clocks and $\mathcal{M}$-rods. In $\mathcal{M}$ the two kinds of clocks and rods behave the same. Indeed, metroclocks and metrorods are designed so that this will happen. But this need not happen in other spacetimes.

\footnotetext{
${ }^{1}$ E.A. Desloge, Foundations of Physics 19, 1191 (1989).

${ }^{2}$ R.F. Marzke and J.A. Wheeler, in Gravitation and Relativity, H.Y. Chiu and W.F.Hoffman, eds. (Benjamin, New York, 1964)

${ }^{3} \mathrm{H}$. Reichenbach, Axiomatization of the Theory of Relativity, University of California Press, 1969, pp. 14, 76, 90.
} 
Let $\mathcal{N}$ be Newtonian spacetime, with an absolute time, the Galilean transformation between inertial frames, and an ether frame in which the speed of light is isotropic. Let $\mathcal{N}^{\prime}$ be the spacetime obtained from $\mathcal{N}$ by restricting the speed of particles in the ether frame to less than that of light.

Consider any inertial frame of $\mathcal{M}$ and the ether frame of $\mathcal{N}^{\prime}$. The worldlines of light and free particles are the same from the viewpoint of these frames. Thus the nonmetric structures of $\mathcal{M}$ and $\mathcal{N}^{\prime}$ are isomorphic.

It follows that the nonmetric structure of $\mathcal{M}$ contains no information about a property of $\mathcal{M}$ which is not also a property of $\mathcal{N}^{\prime}$. In particular, the nonmetric structure of $\mathcal{M}$ contains no information about time dilation or length contraction of $\mathcal{M}$-clocks and $\mathcal{M}$-rods. Nor does it contain any information about a universal light speed. These conclusions hold independently of any theorems proved, any definitions made, or any experimental results obtained about the nonmetric structure.

If we lived in $\mathcal{N}^{\prime}$, a metrosphere would behave exactly as it does in $\mathcal{M}$, since the nonmetric structures of the two spacetimes are isomorphic. In particular, metroclocks and metrorods would exhibit time dilation and length contraction. But we would not say that "the definition of the metrosphere leads to the usual kinematic results of special relativity". For these results are not true in $\mathcal{N}^{\prime}$ ! 